http://jmscr.igmpublication.org/home/ ISSN (e)-2347-176x ISSN (p) 2455-0450

crossref DOI: https://dx.doi.org/10.18535/jmscr/v7i8.79

\title{
A study of frequency domain analysis of heart rate variability in newly diagnosed hypertensives
}

\author{
Author \\ Amit Tak \\ Department of Physiology, SMS Medical College, Jaipur, India \\ Corresponding Author
}

Amit Tak

\begin{abstract}
Background: Hypertension is one of the most preventable risk factor associated with premature death worldwide. Blood pressure regulation involves multiple mechanisms with autonomic nervous system has a prominent role. The present study focus on the early changes during pathogenesis of essential hypertension in autonomic nervous system. The data acquisition includes short term ECG for 5 minutes from hypertensives and controls with standard protocols. The frequency domain analysis has been done to compare the two groups.

Study Objective: The aim of the study was to compare frequency domain measures of heart rate variability in newly diagnosed essential hypertensives with controls.

Design: Observational analytical case control study.

Setting: The study was conducted in the Department of Physiology, SMS Medical College, Jaipur

Sample / Population: Simple random sampling was done. Seventy newly diagnosed hypertensives from Medical OPD of SMS Hospital, Jaipur and seventy normotensives from employees of SMS Medical College, Jaipur were enrolled in the study.

Duration of Study: April 2017 to November 2018.

Results: In frequency-domain analysis, power in high frequency [HF (ms2)] and [HF (nu)], which reflects parasympathetic activity, were significantly less in newly diagnosed hypertensive subjects while $L F(n u)$ and $L F / H F(\%)$, which reflects sympathetic activity, were significantly higher in newly diagnosed hypertensive subjects as compared to normotensives.

Conclusion: These findings suggest that heart rate variability is reduced in subjects with newly diagnosed essential hypertension and the parasympathetic inhibition and sympathetic activation is present in the early stages of essential hypertension.

Keywords: heart rate variability, hypertension, frequency-domain analysis.
\end{abstract}

\section{Introduction}

Essential (primary or idiopathic) hypertension is the most common type of hypertension, affecting 95\% of all cases of hypertension, where a specific underlying disorder causing the elevation of blood pressure cannot be identified. ${ }^{[1]}$ Recommended criteria for the diagnosis of hypertension is average (of two or more seated blood pressure readings during each of two or more outpatient visits) clinic blood pressures of $>140 / 90 \mathrm{mmHg} .{ }^{[1]}$ Hypertension is the most important preventable risk factor for premature death worldwide. 
Hypertension doubles the risk of cardiovascular diseases, including coronary heart disease, congestive heart failure, ischemic and haemorrhagic stroke, renal failure, and peripheral arterial disease. ${ }^{[1]}$ Hypertension is directly responsible for $57 \%$ of all stroke deaths and $24 \%$ of all coronary heart disease deaths in India. ${ }^{[2]}$. This correlation is more robust with systolic than with diastolic $\mathrm{BP}^{[3]}$. This fact is important because hypertension is a controllable disease and a $2 \mathrm{mmHg}$ population-wide decrease in BP can prevent 151,000 stroke and 153,000 coronary heart disease deaths in India ${ }^{[4]}$.

As Blood Pressure is a product of Cardiac Output and Total Peripheral Resistance, both parameters are modulated by two limbs of autonomic nervous system. The correlates of the autonomic activity on heart can be captured by heart rate variability. The Fourier Transform of the RR tachogram reflects the spectral content of the signals. It has been shown the high frequency correlates with high vagal activity and low frequency has the contribution of both sympathetic and parasympathetic activity.

\section{Material and Methods}

The study consists of two groups: patients with newly diagnosed hypertension and without hypertension in the age group of 30 to 50 years. The patients were recruited from the Medical OPD of SMS Hospital, Jaipur and normotensives were recruited from the employees of the SMS Medical College, Jaipur. Seventy patients with confirmed hypertension (systolic blood pressure $>140 \mathrm{mmHg}$ or diastolic pressure > $90 \mathrm{mmHg}$ ) who had never been treated with any hypertensive medication, were the study group and healthy age and sex matched seventy normotensive subjects constitute the control group.

The exclusion criteria were diabetes, secondary hypertension, obesity (BMI>30), known history of acute and chronic illness, patients taking any drug known to affect autonomic nervous system, smokers and alcoholics, and uncooperative subjects. The ethical clearance was obtained from the Ethics Committee of the College. Informed written consent was obtained from all participants. Subjects were instructed to avoid tea, coffee and food at least 2 hours before start of the recording. The recording was taken between 09:00 AM and 12:00 PM. All the participants were subjected to anthropological measurements, that include height, weight, hip circumference and waist circumference. Detailed history and general physical examination was conducted to rule any cause other than essential hypertension. Blood pressure was recorded with automated digital device to avoid manual error.

The ECG signals for heart rate variability (HRV) were recorded with RMS Polyrite D (version 1.0) after supine rest for 15 minutes. The resting ECG was recorded for five minutes at sampling frequency $256 \mathrm{~Hz}$. The discrete ECG time series consists of P-QRS-T waves. RR intervals are calculated from this signal and another series of signal is constructed that consists of RR intervals called tachogram. The Power Spectral Density of the tachogram was obtained and the frequency domain measures were calculated that includes: the power in high frequency (HF) band $(0.15$ $0.40 \mathrm{~Hz})$; power in low frequency $(\mathrm{LF})$ band $(0.04$ $0.15 \mathrm{~Hz}$ ), power in high frequency (HF)band (normalized unit), power in low frequency (LF) band (normalized unit) and LF/HF ratio. The above computation is carried out using Kubois Software.

\section{Statistical Analysis}

The general parameters and frequency domain parameters were compared between newly diagnosed cases of hypertension and normotensives. Descriptive statistics involve the quantitative data which was expressed in mean \pm SD and compared using unpaired Students t-test and qualitative data was expressed in proportions and compared using z-test. A p-value of $<0.05$ was considered statistically significant. Data was analyzed using Microsoft Excel 2010. 


\section{Results}

General Characteristics

General characteristics are shown in Table 1. There was no significant difference of means of age between two populations. Similarly, there is no significant difference of proportions in gender between two populations. However, body mass index (BMI), waist hip ratio (WHR), systolic blood pressure (SBP), diastolic blood pressure (DBP), and heart rate show significant difference of means between the two populations.

Table 1: Mean \pm SD values of general parameters of the study population

\begin{tabular}{|c|c|c|c|c|c|}
\hline & $\mathbf{N}$ & Group & Mean \pm SD & $\begin{array}{c}\text { Difference } \\
\text { of Means }\end{array}$ & $P$ value \\
\hline \multirow{2}{*}{ Age (years) } & 70 & Case & $43.1 \pm 4.15$ & \multirow{2}{*}{0.10} & \multirow{2}{*}{$>0.05(\mathrm{NS})$} \\
\hline & 70 & Control & $43.0 \pm 3.0$ & & \\
\hline \multirow{2}{*}{ BMI $\left(\mathrm{kg} / \mathrm{m}^{2}\right)$} & 70 & Case & $26.39 \pm 2.69$ & \multirow{2}{*}{1.38} & \multirow{2}{*}{$<0.05(\mathrm{~S})$} \\
\hline & 70 & Control & $25.01 \pm 2.46$ & & \\
\hline \multirow{2}{*}{ Waist Hip ratio } & 70 & Case & $0.98 \pm 0.14$ & \multirow{2}{*}{0.07} & \multirow{2}{*}{$<0.05(\mathrm{~S})$} \\
\hline & 70 & Control & $0.91 \pm 0.01$ & & \\
\hline \multirow{2}{*}{ SBP (mmHg) } & 70 & Case & $152.31 \pm 5.03$ & \multirow{2}{*}{22.31} & \multirow{2}{*}{$<0.05(\mathrm{~S})$} \\
\hline & 70 & Control & $130.00 \pm 4.00$ & & \\
\hline \multirow{2}{*}{ DBP (mmHg) } & 70 & Case & $88.70 \pm 4.24$ & \multirow{2}{*}{9.70} & \multirow{2}{*}{$<0.05(\mathrm{~S})$} \\
\hline & 70 & Control & $79.00 \pm 4.00$ & & \\
\hline \multirow{2}{*}{$\begin{array}{l}\text { Heart Rate } \\
\text { (bpm) }\end{array}$} & 70 & Case & $86.09 \pm 5.63$ & \multirow{2}{*}{9.46} & \multirow{2}{*}{$<0.05(\mathrm{~S})$} \\
\hline & 70 & Control & $76.63 \pm 7.9$ & & \\
\hline
\end{tabular}

Frequency domain measures

The difference of means of power in the high frequency band $\left[\mathrm{HF} \quad\left(\mathrm{ms}^{2}\right)\right], \quad[\mathrm{HF}(\mathrm{nu})]$ are significantly reduced $(\mathrm{P}$ value $<0.05)$ in new diagnosed hypertensives. The difference of means of power in low frequency band (normalized unit) $[\mathrm{LF}(\mathrm{nu})]$ and $\mathrm{LF} / \mathrm{HF}$ ratio are significantly increased ( $\mathrm{P}$ value $<0.05)$ in the newly diagnosed hypertensives. The difference of means of Power in low frequency band $\left(\mathrm{ms}^{2}\right)\left[\mathrm{LF}\left(\mathrm{ms}^{2}\right)\right]$ has shown no significant differences ( $P$ value $>0.05$ ) of means between two populations. The results of frequency domain parameters are shown in the Table 2.

Table 2: Mean \pm SD of Frequency Domain Measures of Heart Rate Variability

\begin{tabular}{|c|c|c|c|c|c|}
\hline & $\mathbf{N}$ & Group & Mean \pm SD & Difference of Means & P value \\
\hline $\mathrm{LF}\left(\mathrm{ms}^{2}\right)$ & 70 & Case & $637.66 \pm 568.03$ & \multirow{2}{*}{62.16} & \multirow{2}{*}{$>0.05(\mathrm{NS})$} \\
\hline & 70 & Control & $699.82 \pm 463.51$ & & \\
\hline LF(nu) & 70 & Case & $78.34 \pm 10.94$ & \multirow{2}{*}{25.10} & \multirow{2}{*}{$<0.05(\mathrm{~S})$} \\
\hline & 70 & Control & $53.24 \pm 9.64$ & & \\
\hline $\mathrm{HF}\left(\mathrm{ms}^{2}\right)$ & 70 & Case & $165.26 \pm 203.77$ & \multirow{2}{*}{447.73} & \multirow{2}{*}{$<0.05(\mathrm{~S})$} \\
\hline & 70 & Control & $612.99 \pm 421.19$ & & \\
\hline HF(nu) & 70 & Case & $21.57 \pm 10.87$ & \multirow{2}{*}{24.96} & \multirow{2}{*}{$<0.05(\mathrm{~S})$} \\
\hline & 70 & Control & $46.53 \pm 9.60$ & & \\
\hline LF/HF Ratio & 70 & Case & $5.00 \pm 3.14$ & \multirow{2}{*}{3.73} & \multirow{2}{*}{$<0.05(\mathrm{~S})$} \\
\hline & 70 & Control & $1.27 \pm 0.48$ & & \\
\hline
\end{tabular}

Table 2 exhibits significant differences in means of $\mathrm{LF}(\mathrm{nu}), \mathrm{HF}\left(\mathrm{ms}^{2}\right), \mathrm{HF}(\mathrm{nu})$ and LF/HF Ratio and no significant difference of means of $\operatorname{LF}\left(\mathrm{ms}^{2}\right)$.

\section{Discussion}

The modulatory roles of the dual limbs of autonomic nervous system namely parasympathetic nervous system and sympathetic nervous system in newly diagnosed hypertensives are measured in the present study. The results showed the power in high frequency band $\left(\mathrm{ms}^{2}\right)$ and high frequency band (normalized unit) are significantly reduced. This correlates with the decreased parasympathetic activity during the 
early phase of development of essential hypertension. Similarly, the power in low frequency band (normalized unit) and LF/HF ratio were significantly increased that correlates with the sympathovagal imbalance in the early phase of development of essential hypertension.

Autonomic Nervous System dysfunction is thought to play a significant role in the pathogenesis of essential hypertension. The Autonomic Nervous System modulates the cardiovascular system via two limbs: the Sympathetic and Parasympathetic Nervous System. Many studies correlate the Total Power in high frequency band HF) with parasympathetic tone (Eckberg 1983, Fouadte al 1984, Rimoldi et al 1990), but few studies are against to this view (Kollai and Mizsei, 1990).

Similarly, many studies correlate Power in low frequency (LF) with sympathetic nervous activity ( Rimoldi et al, 1990; ul et al, 1990; Malliani et al, 1991) but many studies contradicted this view (Pagani et al. 1986; Vibyral 1990; Eckbery1997) showed LF also influenced by vagal activity.

In a study of 91 subjects Guzzetti et al (1988) suggested increase in sympathetic activity and reduced parasympathetic activity in essential hypertensive subjects. He found LF was greater and HF was lower in hypertensives as compared to normotensives ${ }^{[5]}$.

In a study conducted by $\mathrm{Wu}$ et al (2013) 1,638 non-hypertensive subjects included and short term HRV ( 5 min) were conducted and followed up for 7 years. Incident hypertension was determined by blood pressure status. In a multivariate model $\mathrm{LF} / \mathrm{HF}$ ratio and $\mathrm{HF}$ were independently associated with incident hypertension. In the present study, increased $\mathrm{LF} / \mathrm{HF}$ ration and decreased $\mathrm{HF}$ is associated with incident hypertension. ${ }^{[6]}$

Study conducted by Julius S et al (1991) quoted that increase sympathetic drive and parasympathetic inhibition are associated with patients with borderline hypertension. This state is also characterized by high heart rate, high cardiac output and normal vascular resistance (hyperkinetic state). In contrast to this established hypertension is associated with normal cardiac output, increased vascular resistance and no signs of increased in sympathetic drive. This mechanism of hemodynamic shift can be understood. Cardiac output returns to normal values and the beta adrenergic receptors downregulate and stroke volume decreases. The high blood pressure increases vascular hypertrophy, that leads to increased vascular resistance. The mechanism of change of sympathetic drive from high to normal can be explained by "bloodpressure seeking" properties of the brain. In hypertension, the blood pressure needs to be maintained at higher levels, as decrease progresses the vascular hypertrophy causes increased vascular resistance. The increase in sympathetic drive found in hypertension is still unsolved. The reason may include other factors as BMI, hyperlipidemia, hyper-insulinemia that are associated with this disease ${ }^{[7]}$.

Goit RK et al (2016) conducted a study in which they took 120 newly diagnosed hypertensives subjects and 120 controls. they showed high frequency $\left[\mathrm{HF}\left(\mathrm{ms}^{2}\right)\right]$ and $[\mathrm{HF}(\mathrm{nu})]$ which reflects parasympathetic activity, was significantly less in hypertensive subjects while $\mathrm{LF}(\mathrm{nu})$ and $\mathrm{LF} / \mathrm{HF}$ (\%) which reflects sympathetic activity, were comparable between the groups. There findings suggests early parasympathetic inhibition in the development of hypertension ${ }^{[8]}$. This view contrast with our study, where both parasympathetic activity decreased and sympathetic activity increased in newly diagnosed cases of hypertension.

\section{Conclusion}

These findings suggest in subjects with newly diagnosed essential hypertension and the parasympathetic inhibition and sympathetic activation is present in the early stages of essential hypertension. The inconsistencies of the results in various studies may be due to multifactorial causes of the development of the hypertension. Multiple factors like sex, race, whether, BMI, 
heart rate can influence the heart rate variability measures independently. This is one of the reason of non-standardization of the heart rate variability parameters.

\section{References}

1. Kotchen TA, Hypertensive Vascular Disease: Harrison's Principles of Internal Medicine, $18^{\text {th }}$ Edition New York : Tata McGraw Hill,2012 pp 2042-59.

2. Raghupathy Anchala, Nanda K. Kannuri, Hira Pant, Hassan Khan, Oscar H. Franco, Emanuele Di Angelantonio, and Dorairaj Prabhakaran Review: Hypertension in India : a systematic review and metaanalysis of prevalence, awareness, and control of hypertension. Journal of Hypertension;.(2014) 32:1170-1177.

3. Cutler JA. High blood pressure and endorgan damage. J Hypertens. 1996;14(suppl 6):S3-S6.

4. Gupta R; Trends in hypertension epidemiology in India ; Journal of Human Hypertension (2004) ; $18: 73-78$.

5. Guzzetti S, Piccaluga E, Casati R, Cerutti S, Lombardi F, Pagani M, Malliani A. Sympathetic predominance in essential hypertension: a study employing spectral analysis of heart rate variability. J Hypertens. 1988 Sep;6(9):711-7

6. Ernst G, Heart Rate Variability, Springer 2014,pp 27-47, 51-103,119-126,188-193.

7. Julius S. Autonomic nervous system dysregulation in human hypertension. American Journal of Cardiology. 1991 Apr 22;67(10):B3-7.

8. Goit RK, Ansari AH. Reduced parasympathetic tone in newly diagnosed essential hypertension. Indian Heart Journal. 2016 Mar 1;68(2):153-7. 